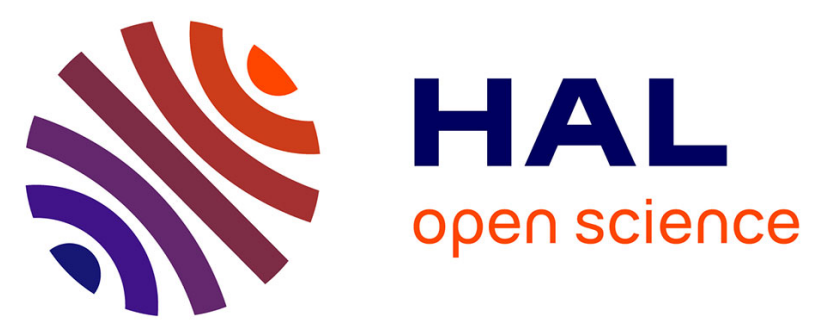

\title{
In elderly Caucasian women, younger facial perceived age correlates with better forearm skin microcirculation reactivity
}

Christelle Guéré, Armelle Bigouret, Alex Nkengne, Katell Vié, Anthony Gélis, Joshua Dulong, Jérôme Lamartine, Bérengère Fromy

\section{To cite this version:}

Christelle Guéré, Armelle Bigouret, Alex Nkengne, Katell Vié, Anthony Gélis, et al.. In elderly Caucasian women, younger facial perceived age correlates with better forearm skin microcirculation reactivity. Skin Research and Technology, 2021, 10.1111/srt.13080 . hal-03406724

\section{HAL Id: hal-03406724 https://hal.science/hal-03406724}

Submitted on 2 Nov 2021

HAL is a multi-disciplinary open access archive for the deposit and dissemination of scientific research documents, whether they are published or not. The documents may come from teaching and research institutions in France or abroad, or from public or private research centers.
L'archive ouverte pluridisciplinaire HAL, est destinée au dépôt et à la diffusion de documents scientifiques de niveau recherche, publiés ou non, émanant des établissements d'enseignement et de recherche français ou étrangers, des laboratoires publics ou privés. 


\title{
In elderly Caucasian women, younger facial perceived age correlates with better forearm skin microcirculation reactivity
}

\author{
Christelle Guéré $^{1}$ | Armelle Bigouret ${ }^{1}$ | Alex Nkengne ${ }^{1}$ | Katell Vié Anthony Gélis $^{2}$ | \\ Joshua Dulong ${ }^{2}$ | Jérôme Lamartine ${ }^{2}$ | Bérengère Fromy²
}

${ }^{1}$ Laboratoires Clarins, Pontoise, France

${ }^{2}$ Laboratoire de Biologie Tissulaire et d'ingénierie Thérapeutique, UMR5305 CNRS - Université Claude Bernard, Lyon 2 Cedex 07, France

\section{Correspondence}

Bérengère Fromy, Laboratoire de Biologie tissulaire et Ingénierie thérapeutique (LBTI), UMR 5305 CNRS/UCBL1, 7 passage du Vercors, 69367 LYON Cedex 07.

Email: berengere.fromy@univ-lyon1.fr

\section{Funding information}

The present study was entirely funded by Clarins (Grant Number 166846).

\begin{abstract}
Background: Visual and molecular changes occurring upon aging are rather well characterized. Still, aging signs show great significant inter-individual variations, and little is known concerning the link between perceived age and cutaneous microcirculation. Materials and Methods: To investigate this point, we recruited Caucasian women in their mid-50's to mid-70's and subsampled women looking older or younger than their age. We studied their facial skin color, as well as their microvascular reactivity to local heating assessed in the forearm skin. We also used skin biopsies from some of these women for gene expression or immunohistochemical analysis.

Results: Clinical and instrumental analysis of skin color revealed that subjects who look 5 years younger differ only by a higher glowing complexion. Our most striking result is that subjects looking 5 years younger than their age present a higher microcirculation reactivity in forearm skin. Transcriptome comparison of skin samples from women looking older or younger than their age revealed 123 annotated transcripts differentially expressed, among which MYL9 relates to microcirculation. MYL9 is downregulated in the group of women looking younger than their real age. Microscopy shows that the labeling of MYL9 and CD31 are altered and heterogeneous with age, as is the morphology of microvessels.

Conclusion: Therefore, assessing generalized vascular reactivity in non-photoexposed skin to focus on the intrinsic aging allows subtle discrimination of perceived age within elderly healthy subjects.

KEYWORDS

aging, microcirculation, MYL9, perceived age, skin, transcriptome
\end{abstract}

\section{1 | INTRODUCTION}

As our societies grow older, skin aging has become an area of increasing research interest. Indeed, skin aging is the most obvious sign of aging. In this context, perceived age, the age someone is visually estimated to have, is socially essential to many individuals, as exemplified by the success of the cosmetic industry, and surgical solutions. It also has implications that go beyond mere aesthetics as it is a robust predictor of mortality and age-related diseases. $^{1-3}$

Jérôme Lamartine and Bérengère Fromy co-last authors.

(c) 2021 John Wiley \& Sons A/S. Published by John Wiley \& Sons Ltd 
As a consequence, many studies have focused on the changes occurring in aging skin. ${ }^{4-7}$ These changes result from two distinct biological processes. The first one is intrinsic aging whatever the skin area, which is genetically determined and occurs with time. The intrinsic aging signs are linked to many structural and molecular changes appearing in all parts of the skin. In particular, the cutaneous microcirculation is altered with age. ${ }^{8}$ The thickness of the basement membranes is decreased, as is the number of perivascular cells, two changes that result in decreased perfusion, and increased capillary fragility. ${ }^{9}$ Several functional studies commonly reported endothelial dysfunction with advanced aging in human and rodent skin. ${ }^{10-14}$

Skin aging can be aggravated by environmental insults (eg, sun exposure, ${ }^{15,16}$ tobacco, ${ }^{17,18}$ pollution ${ }^{19,20} \ldots$, a process known as extrinsic aging that mainly affects the exposed areas of the body: the face and the neck. If each process leads to characteristic signs, both combine to lead to the progressive decay of the skin and visible changes in topographic and color cues: wrinkles, sagging, and uneven skin tone. ${ }^{4,15}$

These aging changes influence one's appearance and show significant interindividual variations that are partially genetically determined. ${ }^{21,22}$ They also have different relative importance on perceived age. ${ }^{22-26}$

All these works pinpoint that inter-individual variation occurs with biological aging. The clinical signs revealing this heterogeneity, and influencing perceived age, are well described. Nevertheless, little is known about the biological markers that underly this heterogeneity and explain the perceived age. If biological age have an influence on skin microcirculation properties, the link between perceived age and skin microcirculation properties have not been previously explored. We hypothesized that women looking younger have better skin microcirculation properties than women looking older. For this purpose, we recruited 55- to 75-year-old subjects who were looking younger or older than their real age. We evaluated and measured skin color parameters that could relate to microcirculation on the subjects of these two groups. We also studied the reactivity of the cutaneous microcirculation in response to local heating in non-photo-exposed skin (forearm) to highlight possible differences on intrinsic aging and performed a microarray analysis to identify molecular markers that would be differentially expressed depending on perceived age.

\section{2 | MATERIALS AND METHODS}

\section{1 | Ethics}

This study was conducted according to Good Clinical Practices and followed the principles of the Declaration of Helsinki. It was approved by an Independent Ethics Committee, the "Comité de Protection des Personnes" of the "Centre Léon Bérard - Sud-Est IV" on May 11th, 2016 and authorized by the "Agence Nationale de Sécurité du Médicament et des Produits de Santé," the competent authority, on June 10th, 2016. Prior to enrolling in the study, written informed consent was obtained from every subject.

\section{2 | Subjects, perceived age groups, treatments, and biopsies sampling}

An initial panel of 104 Caucasian women was recruited. All of them were healthy subjects with age ranging between 55 and 75 years old, a body mass index between 20 and 28 , a skin belonging to phototype II or III on the Fitzpatrick scale. They were non-smokers or have stopped smoking for at least 5 years and without any dermatological pathology affecting the face. The volunteers were asked to avoid sun exposition during the full month before the test in order to prevent self-tanning, which could affect their natural skin complexion and glow. Their living habit toward smoking and sun exposure was also collected and analyzed to ensure similarities among volunteers (Supplementary Information S1). The volunteers did not apply any cosmetic product or makeup on the day of the visit. Four trained experts estimated the perceived age of each subject. One of these experts is a dermatologist, the other 3 are clinical evaluation specialists with more than 12 years of experience, and who had undertaken several training sessions (on average every 2 or 3 weeks) to evaluate the skin parameters clinically. They assessed the perceived age on $4000 \times 6000$ px large standardized pictures (Nikon D7100 equipped with a $60 \mathrm{~mm}$ lens, F/22, 1/125s, ISO-250) of the full face taken under normal diffuse light, with open eyes and a neutral expression (no smile). The estimation of perceived age was performed in two steps. In a first step, experts estimated the perceived age of all subjects based on the sole pictures, without any reference, and focusing on the full skin facial attributes. In a second step, they were told the real age and, upon this knowledge, decided if the subject was looking younger than her age, looking at her age, or looking older than her age. Subjects were further considered in the study if their perceived age given by three of the four experts were consistently younger or older than their real age. Subjects who were looking younger than their age and those looking older than their age were split into different groups, as depicted in Figure 1.

Clinical assessments and instrumental measurements of skin's parameters and microcirculation studies (Figure 1A), were performed on 44 subjects whose average perceived age show at least a 1-year difference from their real age. These volunteers were divided into three groups: one group of 8 women looking at least 5 years younger than their real age, a second group of 14 women looking at least 5 years older than their real age, and the third group containing the 22 last volunteers having a perceived age close to their real age (less than a 5-year difference with their real age). The results of this third group are presented in supplementary information (S1 and S2).

Four millimeter punch skin biopsies were sampled by a clinician in an area of the neck, close to the ear. The subjects selected for these biopsies were the 24 subjects showing the most substantial difference between their perceived age and their real age (Figure 1B). Twelve of them were looking younger than their real age, and 12 more were looking older. In each group, the 6 biopsies from subjects showing the largest difference between perceived and real age were used for microarray studies, and the 6 remaining biopsies were used for immunohistochemical analysis. 
(A) 04 Caucasian women 55 to 75 years old

Evaluation of perceived age by 4 trained experts 3 -step method \& consensus of at least 3 experts out of the

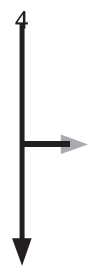

60 Caucasian women without a difference between their perceived age and their real age Excluded from the study

44 Caucasian women

with a difference between their perceived age and their real age

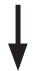

\section{8 women looking at least 5 years younge \\ 14 women looking at least 5 years older}

(B)

104 Caucasian women

55 to 75 years old

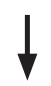

Evaluation of perceived age by 4 trained experts 3-step method \& consensus of at least 3 experts out of the 4

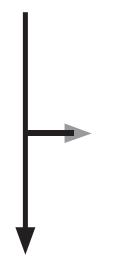

60 Caucasian women without a difference between their perceived age and their real age Excluded from the study

44 Caucasian women

with a difference between their perceived age and their real age

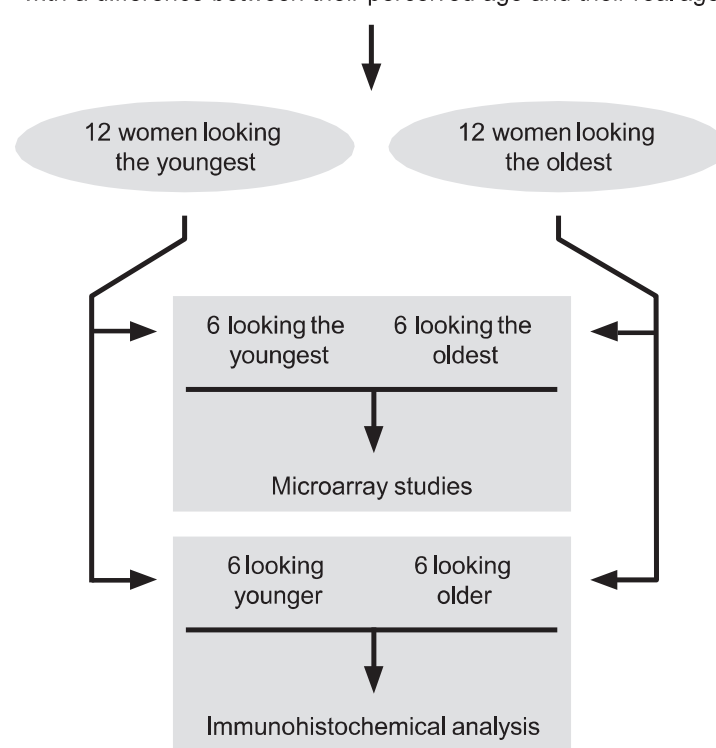

FIGURE 1 Scheme of subjects' selection and of their inclusion for: A, The clinical and instrumental assessment of facial skin as well as for the study of the microcirculation reactivity, B, The microarray studies, and the immunohistochemical analysis

\subsection{Clinical assessments and instrumental measurements of facial skin color}

The skin color of each subject was clinically graded by three trained experts using standardized full-face pictures. The parameters studied were the pink complexion, the beige complexion, the uniformity of the complexion, and the glowing complexion, this last parameter corresponding to the global luminosity/reflection that emanates from the entire face. These parameters were all evaluated on a 0 (none/not at all) to 15 (very) unstructured scale. Skin color (L*a*b*) was also instrumentally measured using a CM700-d spectrocolorimeter (Konica Minolta) on an $8 \mathrm{~mm}$ diameter zone of the cheek.

\subsection{Assessment of the reactivity of cutaneous microcirculation (forearm skin)}

Cutaneous microcirculation was studied by analyzing reactivity of the skin microcirculation in response to local heating in the forearm skin, which is of great interest in providing information on the underlying mechanisms of non-photo-exposed skin to focus on internal aging. ${ }^{27}$ The experiment started after a 30 -minute acclimation in a temperature- and humidity-controlled environment $\left(22 \pm 2^{\circ} \mathrm{C}\right.$ and 35 to $55 \%$ hygrometry). During these 30 minutes, the subjects were in a semi-recumbent position, and the temperature of the skin was stabilized between 33.5 and $35^{\circ} \mathrm{C}$. Skin blood flow was recorded on the volar surface of the forearm using Laser Doppler probe (457$\mathrm{PI}$, Perimed) in response to local heating of $42^{\circ} \mathrm{C}$ thanks to a Temp Unit (PF 5020, Perimed) as previously reported. ${ }^{14}$ Data collection included a one-minute baseline period before the onset of local heating. Local heating and recording were continued for 20 minutes. The recording included measuring blood flow at the site of heating, blood flow outside of the heated zone ( 3 to $5 \mathrm{~cm}$ away), and measurement of cutaneous temperature (440 sensors; Perimed) to check the stability of the skin temperature.

To ensure that results are indeed due to skin microcirculation's sole reactivity, we performed several controls during the acclimation period. The first one is a cutaneous perception threshold test on the internal face of each forearm of all subjects using Von Frey monofilaments to detect possible impaired touch perception, previously 
reported with advancing age. ${ }^{28}$ We also monitored non-invasively the systolic arterial blood pressure, the diastolic arterial blood pressure, and the heart rate, comparing results before and after the microcirculation recordings.

\section{5 | Gene expression profiling (neck skin)}

After temporary storage of skin biopsies in RNAlater ${ }^{\circledR}$ (Thermo Fischer Scientific) at $4^{\circ} \mathrm{C}$, RNAs were rapidly extracted using mirVana ${ }^{\circledR}$ isolation kit (Thermo Fischer Scientific) according to the manufacturer's instructions with only one modification: biopsies were crushed in the lysis buffer using a plastic RNase free pellet pestle ${ }^{\circledR}$ $\left(\right.$ Kimble $\left.^{\circledR}\right)$. Microarray processing and data analysis were performed at the ProfileXpert core facility (Lyon, France) using a high-density oligonucleotide array (Human Transcriptome Array GeneChip HGST 2.0, Affymetrix). RNA (100 ng) was reverse-transcribed, preamplified, and labeled with the GeneChip WT PLUS reagent kit (Affymetrix). Amplified cDNA quantification was performed with a NanoDrop (Thermo Fischer Scientific) and quality checked with the Agilent 2100 Bioanalyzer. Fragmentation of cDNA $(5.5 \mu \mathrm{g})$ and hybridization were performed following Affymetrix protocols. The arrays were read with a confocal laser (GeneChip Scanner 3000 7G, Affymetrix). Then, CEL files were generated using the Affymetrix GeneChip Command Console (AGCC) software 3.0. The obtained data were normalized with Affymetrix Expression Console software v1.3.1 using the RMA statistical algorithm. Genes differentially expressed were identified by Student's test (unequal variance) comparing groups, including triplicates samples. Only genes with $P<.05$ and a fold change of at least 1.3 were considered. Functional annotation of these differentially expressed genes was performed using the DAVID functional annotation tool (https://davis.ncicrf.org). The raw and normalized data have been deposited into the GEO database and are available under the accession number: GSE134204.

For MYL9 RT-qPCR, one reverse transcription per biopsy was performed using the PrimeScript RT Reagent kit (Takara). We also performed two technical duplicates of qPCR using a SYBR Premix ExTaq II (Takara) on an AriaMx Real-Time PCR system (Agilent Genomics) using the following oligonucleotides: MYL9f 5'-CAC-CA G-AAG-CCA-AGA-TGT-CC-3' \& MYL9r 5'-TTG-AAA-GCC-TCC-TTA -AAC-TCC-3'. Results were normalized to the expression level of the TBP (TATA Binding Protein) gene. Relative quantification was calculated using the $2-\Delta \Delta C_{t}$ quantification method.

\section{6 | Histological and immunohistochemical analyses of the biopsies (neck skin)}

Skin biopsies were fixed in buffered formalin for 24 hours, embedded in paraffin, and cut into $5 \mu \mathrm{m}$-thick transversal sections. After deparaffination, the sections were used for immunohistochemical analysis of CD31 (Platelet and Endothelial Cell Adhesion Molecule 1) or MYL9 (Myosin Light Chain 9). Citrate and microwave unmasking was performed for MYL9 labeling. CD31 was labeled using a rabbit polyclonal anti-CD31 antibody (1:80, Ref. M0821, Dako) and MYL9 using a rabbit polyclonal anti-MYL9 antibody (1:100, Ref. HPA059197; Sigma-Aldrich). All incubations with primary antibodies were performed overnight at $4^{\circ} \mathrm{C}$ in PBS buffer supplemented with $3 \%$ of BSA. After inhibition of endogenous peroxidase by hydrogen peroxide, slides were incubated with the secondary antibody, a mouse anti-rabbit IgG, coupled with peroxidase (K4003, Dako) and allow to incubate with its substrate: diaminobenzidine (K3468, Dako). Mayer's hematoxylin was used as counterstaining. Slides were finally analyzed using a Leica DM2000 optical microscope equipped with a Leica DFC420C digital camera and the image acquisition tool LAS v4.2 (Leica). The number of vessels positive for CD31 and MYL9 was counted for each section, and the density was expressed as vessel number by $\mu \mathrm{m}^{2}$.

\section{7 | Statistical analysis}

Results are expressed as mean \pm standard deviation. Normal distribution of data was assessed using a Shapiro-Wilk test $(\alpha=0.01)$. Real and perceived ages were compared using the unpaired $t$ test. Unpaired t test was also used to analyze the statistical relevance of expression changes for the microarray experiment and RT-qPCR results. For the cardiovascular and mechanical sensitivity controls of the microcirculation experiments, comparison of results between the beginning and the end of the experiment within a group of similar perceived age were performed using the paired $t$ test. Results from clinical scoring, instrumental measurements, and microcirculation reactivity-including controls-were compared using unpaired $t$ test and analysis of covariance (ANCOVA) with the real age value as a covariate. Unlike the $t$ test, which does not differentiate the impact of the real age and the perceived age of the two groups, the ANCOVA test makes it possible to remove, statistically, the indirect effect of the real age in order to analyze the impact of perceived age. Results were considered significant for $P<.05$.

\section{3 | RESULTS}

\section{1 | Clinical and instrumental studies of facial skin color show no differences between subjects looking five years younger than their age and those looking five years older}

Clinical and instrumental characterization of facial aging was realized on subjects whose average perceived age shows, at least, a 5year difference with their real age. Such criteria led to a first group of 8 women looking 5 years younger than their real age. Their real age is $68.75 \pm 3.01$ years old while their perceived age is $62.44 \pm 2.85$; this group shows a highly significant age difference $(P=.0008)$ of $-6.31 \pm 1.47$ years between real age and perceived age. The second group corresponds to women looking 5 years older than their age. 
It includes 14 women whose average real age is $59.57 \pm 3.01$, while their perceived age is $67.43 \pm 3.74$. This is a $+7.86 \pm 1.94$ years age difference that is highly significant $(P<.0001)$. The perceived age of the two groups is different $(P=.0038)$, as is their real age $(P<.0001)$. It is also to be noted that the subjects looking older than their age are, in fact, younger than the group of subjects looking younger than their age. Thus the real age of subjects impacts the measures. As shown in Table 1, P-values issued from unadjusted unpaired $t$ test and from ANCOVA (age-adjusted test) are different. We choose the ANCOVA test because we want to focus on the perceived age.

To initially characterize these two groups' subjects, we studied their facial skin color since it might be related to their cutaneous microcirculation. Results from clinical scoring (Table 1) show that the glow complexion is higher $(+30.6 \%, P=.002)$ for subjects looking younger than for subjects looking older than their age. The other clinically evaluated parameters-the pink complexion, the beige complexion, and the uniformity of the complexion-are similar in both perceived age groups.

This absence of difference is also observed when performing instrumental measurements (Table 2): L*a*b* color parameters are similar for subjects looking younger and for those looking older than their real age.

\subsection{Subjects looking five years younger than their real age show higher microcirculation reactivity than those looking five years older than their age}

To find if a relationship exists between perceived age and generalized cutaneous microcirculation, we analyzed the reactivity of the skin microcirculation in response to local heating in a non-photoexposed skin to focus on the internal aging (forearm skin). Basal skin blood flow and basal cutaneous vascular conductance (calculated as skin blood flow/systolic arterial blood pressure) were not different between the group of subjects looking younger than their real age and the group looking older (Figure 2, Table 3). In contrast, differences were found between the two groups of subjects regarding the skin's microcirculation reactivity in response to local heating (Figure 2, Table 3). In both groups, local heating at the level of the forearm induces biphasic vasodilation with a fast response peak followed by a plateau that reaches a maximum value after 20 minutes. While the peak occurs at the same time in both groups, the amplitude of the peak is $41 \%$ higher $(P=.027)$ in the group of subjects looking 5 years younger than in the group of subjects looking 5 years older than their real age. In addition, the amplitude of the plateau is $26 \%$ higher $(P=.038)$ in the group of subjects looking younger than in those looking older than their real age.

Results show that the blood flow 3 to $5 \mathrm{~cm}$ away from the heated zone is stable prior, during, and after the experiment and is not different between the two groups (Figure 2). Basal systolic and diastolic arterial pressures are not different between the two groups ( $p$ ANCOVA $=0.961$ ) and remain stable in the two groups through the experiment (Supplementary information S3), indicating cardiovascular stability.

Basal cardiac frequency is also similar in both groups, while it decreases during the experiment (Supplementaryinformation, Table S3), due to the prolonged bed rest in a quiet room. In addition, measures of cutaneous temperature in the vicinity of the heated zone show good thermal stability (Supplementary information S3). Finally, the cutaneous mechanical perception threshold tests revealed no peripheral sensory neuropathy in any subjects (Supplementary information S3).

\subsection{Transcriptome analysis of skin biopsies from women of various perceived age reveals 123 modulated transcripts that are annotated}

To identify molecular markers that are differentially expressed according to perceived age, we analyzed the 12 samples showing the largest difference between their perceived and their real age. This transcriptome analysis included the 6 subjects looking the youngest, their real age being $68.50 \pm 3.02$ years old while the average perceived age given by the experts was $62.67 \pm 2.73$ years old. This group's subjects show a highly significant difference $(P=.0057)$ of $-5.83 \pm 1.20$ years between their real age and their perceived age. The analysis also included the 6 subjects looking the oldest with a real age of $59.00 \pm 2.37$ years old and a perceived age of $68.58 \pm 3.72$ years old, therefore showing a significant age difference of $9.58 \pm 1.59$ years $(P=.0003)$. The real age of the two groups is different $(P=.0001)$, as is their perceived age $(P=.0106)$, and the subjects looking younger are in fact older than the group of subjects looking older.

We performed a comparative transcriptome analysis between skin biopsies from these two groups. Student's t test and fold change

TABLE 1 Clinical scoring of facial skin

\begin{tabular}{|lllll|} 
& $\begin{array}{l}\text { Subjects looking older than } \\
\text { their real age }\end{array}$ & $\begin{array}{l}\text { Subjects looking younger than } \\
\text { their real age }\end{array}$ & $\begin{array}{l}\text { Unpaired t test } \\
(\boldsymbol{P} \text {-value })\end{array}$ & $\begin{array}{l}\text { ANCOVA } \\
(\boldsymbol{P} \text {-value })\end{array}$ \\
\hline Pink complexion & $2.56 \pm 1.45$ & $3.88 \pm 1.02$ & .034 & .958 \\
\hline Beige complexion & $3.45 \pm 1.67$ & $3.38 \pm 1.55$ & .924 & .113 \\
\hline Uniform complexion & $7.39 \pm 1.72$ & $7.62 \pm 1.72$ & .762 & .283 \\
\hline Glowing complexion & $5.03 \pm 0.74$ & $6.57 \pm 1.06$ & .0007 & .002 \\
\hline
\end{tabular}

Note: Results are expressed as mean \pm standard deviation. Complexions are graded on a 0 to 15 unstructured scale. $P$-values are the results of an unpaired $t$ test and an analysis of covariance (ANCOVA) using the real age as a covariate. Results considered significant $(P<.05)$ are in bold. 
TABLE 2 Measured parameters of facial skin color

\begin{tabular}{llll} 
& $\begin{array}{l}\text { Subjects looking older than } \\
\text { their real age }\end{array}$ & $\begin{array}{l}\text { Subjects looking younger than } \\
\text { their real age }\end{array}$ & $\begin{array}{l}\text { Unpaired } t \text { test } \\
(\boldsymbol{P} \text {-value })\end{array}$ \\
\hline $\mathrm{L}^{*}$ color parameter & $62.70 \pm 2.06$ & $61.13 \pm 2.78$ & .146 \\
a* color parameter & $11.93 \pm 1.47$ & $12.44 \pm 1.73$ & .474 \\
\hline$b^{*}$ color parameter & $17.56 \pm 1.69$ & $17.82 \pm 1.36$ & .292 \\
\hline
\end{tabular}

Note: Results are expressed as mean \pm standard deviation. $L^{*}, a^{*}, b^{*}$ values are expressed on a 0 to 100 scale. $P$-values are the results of an unpaired $t$ test and an analysis of covariance (ANCOVA) using the real age as a covariate.

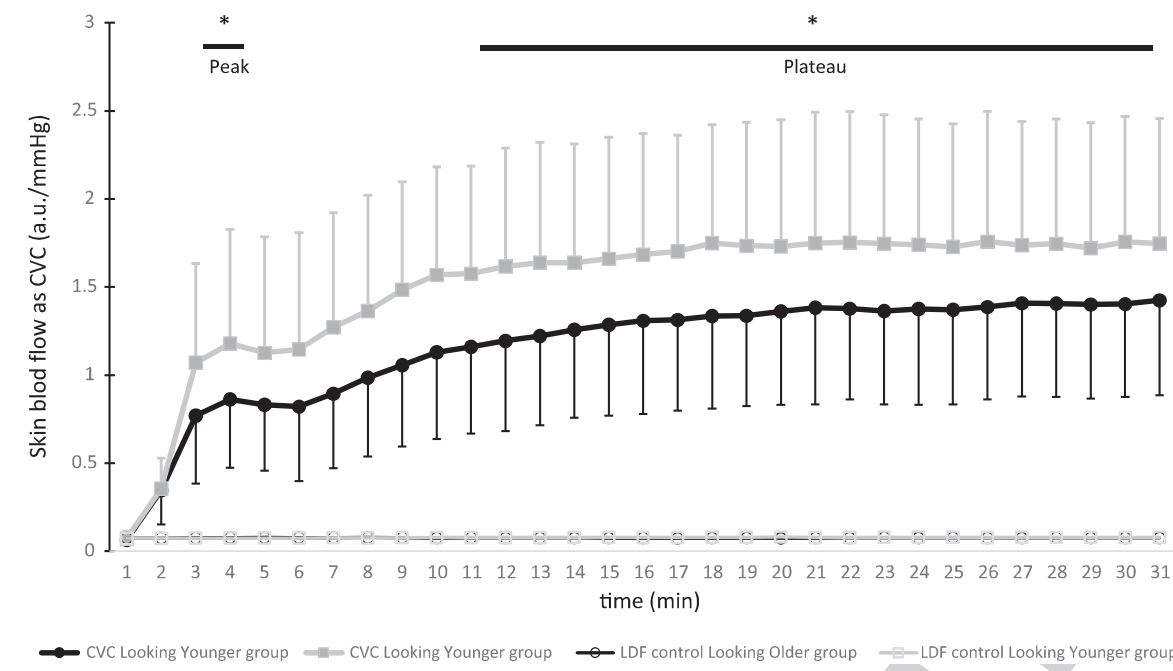

FIGURE 2 Cutaneous blood conductance in response to local heating in the group of subjects looking $5 \mathrm{y}$ older than their real age and in the group of subjects looking 5 y younger than their real age

TAB LE 3 Microcirculation reactivity in forearm skin

\begin{tabular}{lllll} 
& $\begin{array}{l}\text { Subjects looking older than } \\
\text { their real age }\end{array}$ & $\begin{array}{l}\text { Subjects looking younger than their } \\
\text { real age }\end{array}$ & $\begin{array}{l}\text { Unpaired } t \text { test } \\
(\boldsymbol{P} \text {-value })\end{array}$ & $\begin{array}{l}\text { ANCOVA } \\
(\boldsymbol{P} \text {-value })\end{array}$ \\
\hline Baseline value & $7.46 \pm 3.26$ & $9.52 \pm 5.36$ & .272 & .595 \\
\hline Baseline CVC & $0.064 \pm 0.027$ & $0.073 \pm 0.043$ & .576 & .576 \\
\hline Peak CVC & $0.90 \pm 0,11$ & $1.27 \pm 0.23$ & .115 & .027 \\
\hline Plateau CVC & $1.38 \pm 0.15$ & $1.75 \pm 0.26$ & .201 & .038 \\
\hline
\end{tabular}

Note: Results are expressed as mean \pm standard deviation. Skin blood flow is expressed in arbitrary units (a.u.) and cutaneous vascular conductance (CVC) in a.u. $/ \mathrm{mmHg}$. $P$-values are the results of an unpaired $t$ test and an analysis of covariance using the real age as a covariate. Results considered significant $(P<.05)$ are in bold.

(FC) threshold identified 185 transcripts upregulated in biopsies of subjects looking younger than their real age compared to those looking older than their real age (Supplementary Information S4). Eightynine of these sequences correspond to non-annotated transcripts, 5 correspond to microRNAs, and only 87 correspond to annotated mRNA transcripts. Statistical analysis also identified 27 annotated mRNA transcripts, 4 microRNAs, and 54 non-annotated sequences that are downregulated in biopsies of subjects looking younger than their real age compared to those looking older than their real age (Supplementary information S5).

Bio-informatic functional analysis of the 123 annotated transcripts and microRNAs that are modulated reveals only one significantly enriched function: intermediates filaments, keratin type $\left(P=1.1 \times 10^{-5}\right)$. Indeed, four genes involved in the synthesis of type
I keratin (KRT31, KRT33A, KRT33B, KRT43) and two type II keratin synthesis genes (KRT75, KRT85) are repressed in the skin of women looking younger. This might reflect a specific epidermal differentiation status even if these keratins are more specific to hair follicles structures. We also individually examined the other modulated transcripts' putative involvement by direct interrogation of the PubMed database with the gene name and the following keywords: microcirculation, vascular, vessels, and endothelium. This enabled us to identify several modulated transcripts potentially related to vascular functions such as THBS1 encoding Thrombospondin 1 (FC: -1.33 , $P=.021)$, MYL9 encoding Myosin Light Chain 9 (FC: $-1.34, P=.007$ ) and miR-126 (FC: $-1.39, P=.03$ ) a microRNA expressed in vascular endothelial cells. These two genes and the microRNA are downregulated in biopsies of subjects looking younger than their real age. 
We also identified transcripts potentially associated with a vascular function and that are induced in women looking younger than their real age: PTGER3 encoding Prostaglandin E Receptor 3 (FC: 1.44, $P=.02)$ and STRN encoding the Striatin, Calmodulin-Binding Protein (FC: $1.32, P=.03)$.

Among these modulated genes, we particularly focused on MYL9 that is induced upon aging in arterial tissues (Shehadeh et al, 2011). We confirmed by real-time qPCR that MYL9 transcript was repressed by 2 folds ( $1.29 \pm 0.53$ vs. $0.60 \pm 0.29, P=.0183$ - Figure 3 ) in women looking younger than their real age compared to women looking older.

\section{4 | Immunohistochemical labeling of MYL9 and CD31 are highly variable}

To further study MYL9, we performed an immunohistochemical analysis on thin sections of skin biopsies. This analysis was performed on the biopsies of 12 subjects, 6 looking younger and 6 looking older than their real age. The subjects looking younger have a real age of $67.33 \pm 2.58$ years old while their perceived age is $64.42 \pm 2.85$ years old, therefore showing an age difference of $-2.92 \pm 0.79$ years is at the limit of significance $(P=.093)$. The 6 subjects looking older than their real age were $60.67 \pm 3.39$ years old while their perceived age is $67.58 \pm 3.42$; a significant age difference of 6.92 years $(P=.006)$. In the case of these groups of subjects, the real age of the two groups is different $(P=.003)$, but their perceived age is not $(P=.113)$.

The analysis of MYL9 was performed in parallel with that of CD31 (Cluster Differentiation 31 or Platelet Endothelial Cell Adhesion Molecule 1), a protein present at the surface of thrombocytes, monocytes, macrophages, and neutrophils. MYL9 is also present in the intercellular junctions of endothelial cells, therefore, labeling blood vessels. Due to the variability of both labeling (Figure 4), samples were analyzed independently from the perceived age. Indeed, several samples present different degrees of matrix alterations that induce or cause changes in the vascular endothelium or detachment of the perivascular matrix as shown by CD31 labeling, that can combine with more or less developed vasculitis. In addition, CD31 labeling shows that the density, morphology, and distribution of vessels also significantly differ according to the skins analyzed. The number of vessels positive for CD31 was counted for each section and the analysis of these data confirmed a substantial heterogeneity among the different samples (data not shown). In the majority of samples, the vessels present in the dermal matrix are mainly capillaries and venules/arterioles of small diameters. Some samples however show some sections of arterioles/venules of moderate diameter. At the vascular level, MYL9 labeling is also heterogeneous with only a few positive vessels per section. Therefore, the quantification of the labeled vessels was very uncertain and did not show any significant difference among samples (data not shown). Although weak and limited to the deep dermis in most samples, labeling of MYL9 could be observed in the intima, consisting only of endothelial cells, and

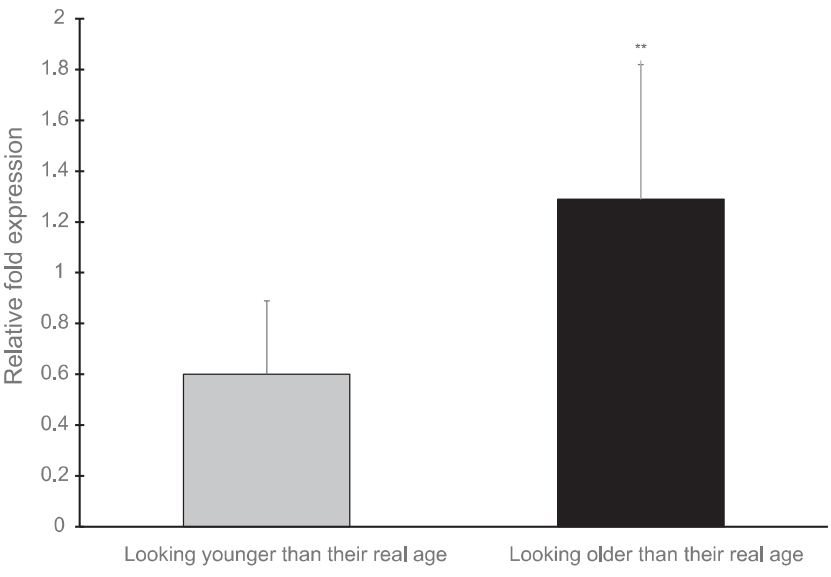

FIGURE 3 RT-qPCR validation of MYL9 transcript downregulation in biopsies from 6 subjects looking older than their real age (in black) compared to biopsies of 6 subjects looking younger than their real age (in grey). MYL9 expression is normalized to the expression of TPB (TATA) Binding Protein, and results are shown as the mean of the $2-\Delta \Delta C_{t} \pm$ standard deviation. $P=.0183^{* *}$ (unpaired $t$ test)

sometimes in the media, composed mainly of smooth muscle cells. In the epidermis, the labeling of MYL9 is mainly cytoplasmic.

\section{I DISCUSSION}

So far, studies focusing on perceived age essentially aimed at identifying key parameters influencing age perception, revealing a prominent role for wrinkling, sagging, and loss of color homogeneity. 22-26,29-31 Our goal was to move one step further and identify biological markers of perceived age in this work. To do so, we focused on a known age-related skin alteration-the deleterious effect of aging on cutaneous microcirculation-reasoning that differences could lead to changes in blood flow and, therefore to discrepancies in the supply of nutrients to the skin that could affect perceived age.

As a first approach to cutaneous microcirculation in women of different perceived ages, we studied facial skin color. Indeed, skin color mainly results from light absorption/reemission by the skin's two main chromophores: melanin, and hemoglobin. ${ }^{32}$ Nevertheless, no differences in instrumental measurements were observed between women looking younger or older than their real age. The only difference we found is that women looking younger than their real age present a higher glowing complexion than those looking older. This glowing complexion does not directly relate to color. Contrary to instrumental measures performed on limited areas of the skin, the glowing complexion is a global parameter that considers the luminosity and the reflection of the skin. It might reflect a better skin health.

Our study's most striking result is the significatively higher microvascular heat-evoked reactivity in a non-photo-exposed skin (forearm) of women looking younger than their real age than of women looking older (assessed in the facial skin). Moreover, the intermediate group with people looking about their age shows a 

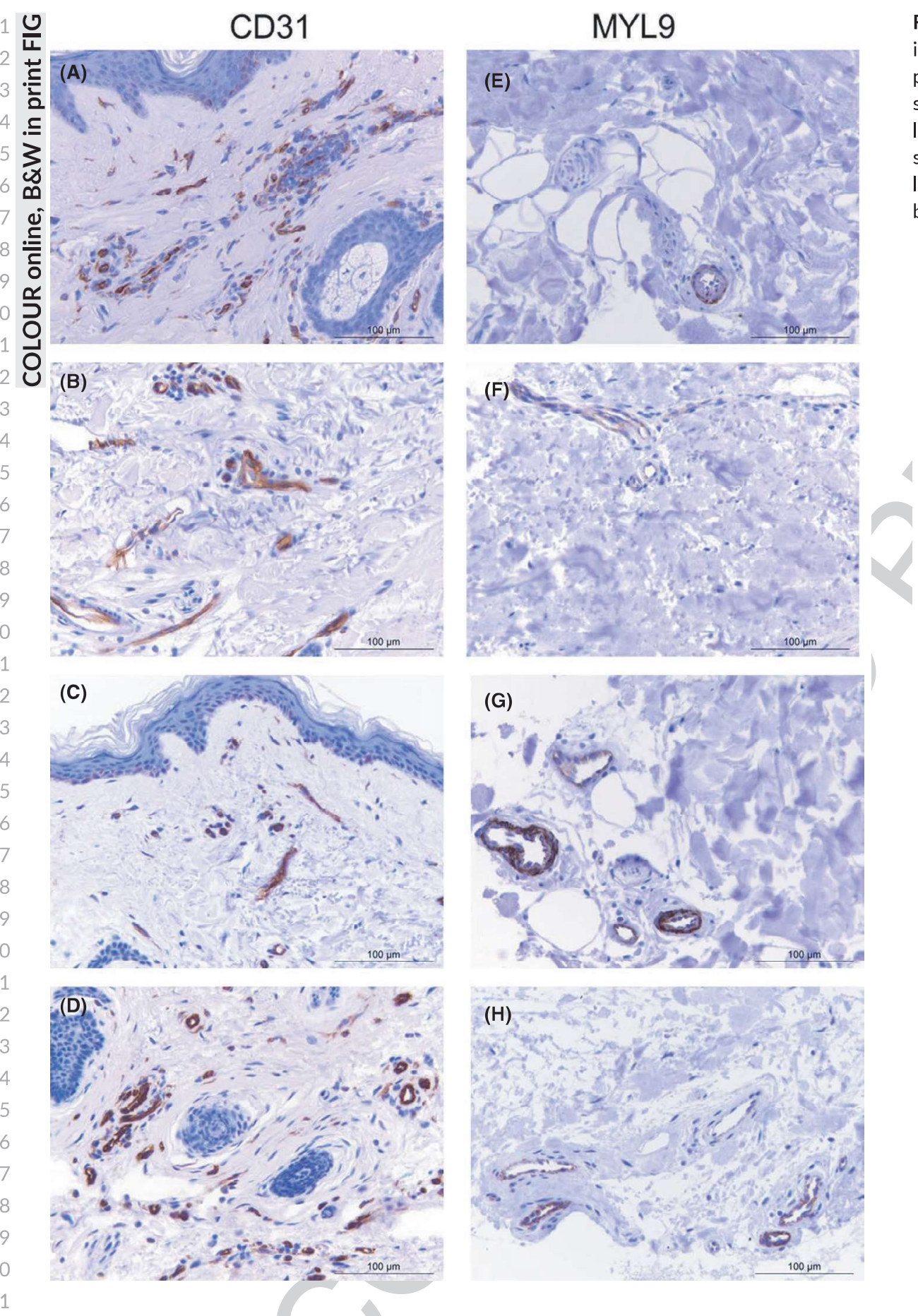

FIGURE 4 Representative microscopy images of the IHC labeling of CD31 (left panel) and of MYL9 (right panel). (A), (E) subject 25 and $(B),(F)$ subject 27 ; subjects looking younger than their real age. (C), (G) subject 15 and (D), (H) subject 18; subjects looking older than their real age. Scale bar: $100 \mu \mathrm{m}$ microvascular reactivity between the 2 others (Supplementary information S2) even if there is no significant difference between the looking older group and "about their age" group results, neither between the looking younger group and the "about their age" group. This result is even more compelling that the women looking younger than their real age are the oldest. Indeed, their average real age is almost 10 years older than that of the group of women looking older than their real age. This result is also specific to the cutaneous microcirculation since monofilament results show no difference in tactile perception, demonstrating the absence of sensory neuropathy. This heat-induced vasodilatation mostly studied in the forearm skin is mediated by neurovascular mechanisms ${ }^{33,34}$ where nerve fibers lead to vasodilation through the activation of downstream second messengers that include NO. ${ }^{35}$ Despite greater sensory afferent nerve densities in facial skin, the initial CVC peak to local heating is similar in non-glabrous and glabrous skin. ${ }^{36}$ This supports that the magnitude of the local heating-mediated vasodilation observed in the forearm skin would have been similar in the facial skin. With aging, vasculature presents reduced NO bioavailability due to decreased synthesis of NO through age-related decreases in L-arginine and $\mathrm{BH} 4$ availability. There would also be increased NO degradation through oxidant stress. Our results demonstrated that, between 55 to 75 years old, women who look younger have a better microcirculatory function in non-photo-exposed area, which exclude the role 
of photo-exposition in this mechanism. These results suggest that maintaining microcirculatory health could participate in maintaining general skin health. Implementing strategies leading to better microcirculatory health and measuring its impact on skin perceived age might be a path of future researches.

The microarray analysis did not provide us any further clue on the underlying mechanisms that would explain the observed difference in the heat-evoked vasodilatation. Globally, it led to the detection of a rather low number of significantly modulated transcripts (123 annotated transcripts including microRNAs), and most of these transcripts exhibited modest fold changes (from 1.3 to 2.18 ) between women looking younger or older than their real age. The high individual variability inside the 2 groups was probably one explanation of this modest transcriptional response. Nevertheless, we identified few modulated transcripts that can be directly or indirectly related to aging or microcirculation. This is the case of the microRNA miR-126, which expression level decreases in the skin interstitial fluids of elderlies while it is secreted by dermal endothelial cells of young skins where it promotes angiogenesis. ${ }^{37}$ Mir-126 is then potentially involved in the skin's vascular function, but the functional consequences of its modulation in total skin samples need to be further clarified.

The other interesting candidate microarray identified is MYL9, a protein that, in rat, increases in the blood vessels of older individuals. ${ }^{38}$ The fate of MYL9 in human aging is not known. However, we can assume that, if similar, repression of this gene in the group of women looking younger than their real age-but chronologically older-makes it a better candidate to be a molecular marker of perceived age and could reflect some specific property of the skin microcirculation in these individuals. Indeed, Shehadeh et al ${ }^{38}$ show that the ectopic expression of MYL9 in rats causes endothelial dysfunction that could explain the increased arterial constriction of aged vasculature. Endothelial dysfunction in the aorta of older rats is also associated with a decreased vasorelaxation due to a decline in $\mathrm{NO}$ and in endothelium-derived hyperpolarizing factor. ${ }^{39,40}$ If these sole elements are not enough to directly link the lower amount of MYL9 in the blood vessels of younger-looking women and their higher microcirculation reactivity, further experiments to explore the role of MYL9 in humans would be of interest.

In conclusion, the most striking characteristic of perceived age we identified is the forearm skin's vascular reactivity, which is improved for subjects looking younger than their real age. Still, the few differences we identified, suggest that the difference between real and perceived age is probably not correlated to strong molecular features. They would also suggest that the skin's response to physical stimulation or external stress might be a better estimator of the heterogeneity of biological aging.

\section{ACKNOWLEDGEMENTS}

The authors would like to thank the Laboratoire Dermscan (Villeurbanne, France) for recruiting the volunteers, performing the clinical assessments and the instrumental measurements, the Novotec company (Bron, France) for histological analysis, and the ProfileXpert genome facility (www.profilexpert.fr) for microarray analysis. The authors also thank Valérie Oréa for her help in performing the cutaneous microcirculation reactivity tests. Finally, they thank Dr Philippe Crouzet, Estium-Concept, for scientific writing services.

\section{CONFLICT OF INTEREST}

$C G, A B, A . N$, and $K V$ are full-time employees of Clarins; a major company specialized in the design, manufacturing, and marketing of cosmetic products.

\section{AUTHOR CONTRIBUTION}

$\mathrm{CG}, \mathrm{AB}, \mathrm{A} . \mathrm{N}$, and $\mathrm{KV}$ designed the research study, analyzed the date and wrote the paper. AG and JD wrote the paper. JL and BF designed the study, performed the research, analyzed the data and wrote the paper.

\section{DATA AVAILABILITY STATEMENT}

The datasets generated during and/or analyzed during the current study are available from the corresponding author on reasonable request.

\section{REFERENCES}

1. Christensen $\mathrm{K}$, lachina $\mathrm{M}$, Rexbye $\mathrm{H}$, et al. "Looking old for your age": genetics and mortality. Epidemiology. 2004;15(2):251-252.

2. Castelo-Branco C, Pons F, Gratacós E, Fortuny A, Vanrell JA, González-Merlo J. Relationship between skin collagen and bone changes during aging. Maturitas. 1994;18(3):199-206.

3. Makrantonaki E, Schönknecht P, Hossini AM, et al. Skin and brain age together: the role of hormones in the ageing process. Exp Gerontol. 2010;45(10):801-813.

4. Glogau RG. Physiologic and structural changes associated with aging skin. Dermatol Clin. 1997;15(4):555-559.

5. Jenkins G. Molecular mechanisms of skin ageing. Mech Ageing Dev. 2002;123(7):801-810

6. Bazin R, Doublet E. Skin Aging Atlas: Volume 1. Caucasian Type Med'Com. 2007.

7. Campisi J, Kapahi P, Lithgow GJ, Melov S, Newman JC, Verdin E. From discoveries in ageing research to therapeutics for healthy ageing. Nature. 2019;571(7764):183-192.

8. Scioli M, Bielli A, Arcuri G, Ferlosio A, Orlandi A. Ageing and microvasculature. Vascular Cell. 2014;6(1):19.

9. Braverman IM, Fonferko E. Studies in cutaneous aging II. The microvasculature. J Invest Dermatol. 1982;78(5):444-448

10. Algotsson A, Nordberg A, Winblad B. Influence of age and gender on skin vessel reactivity to endothelium-dependent and endothelium- independent vasodilators tested with iontophoresis and a laser doppler perfusion imager. J Gerontol Series A: Biol Sci Med Sci. 1995;50A(2):M121-M127.

11. Rossi M, Cupisti A, Mariani S, Santoro G, Pentimone F. Endotheliumdependent and endothelium-independent skin vasoreactivity in the elderly. Aging Clin Exp Res. 2002;14(5):343-346.

12. Tao J. Reduced arterial elasticity is associated with endothelial dysfunction in persons of advancing age Comparative study of noninvasive pulse wave analysis and laser Doppler blood flow measurement. Am J Hypertens. 2004;17(8):654-659.

13. Gaubert ML, Sigaudo-Roussel D, Tartas M, Berrut G, Saumet JL, Fromy B. Endothelium-derived hyperpolarizing factor as an in vivo back-up mechanism in the cutaneous microcirculation in old mice: ageing and endothelium-derived hyperpolarizing factor. J Physiol. 2007;585(2):617-626 
14. Fromy B, Sigaudo-Roussel D, Gaubert-Dahan M-L, et al. Agingassociated sensory neuropathy alters pressure-induced vasodilation in humans. J Investig Dermatol. 2010;130(3):849-855.

15. Jackson R. Elderly and sun-affected skin. Distinguishing between changes caused by aging and changes caused by habitual exposure to sun. Can Fam Physician. 2001;47:1236-1243.

16. Flament F, Bazin R, Laquieze S, Rubert V, Simonpietri E, Piot B. Effect of the sun on visible clinical signs of aging in Caucasian skin. Clin Cosmet Investig Dermatol. 2013;6:221-232.

17. Morita A. Tobacco smoke causes premature skin aging. J Dermatol Sci. 2007;48(3):169-175.

18. Lecas $\mathrm{S}$, Boursier E, Fitoussi $\mathrm{R}$, et al. In vitro model adapted to the study of skin ageing induced by air pollution. Toxicol Lett. 2016;259:60-68.

19. Hüls A, Vierkötter A, Gao W, et al. Traffic-related air pollution contributes to development of facial lentigines: further epidemiological evidence from Caucasians and Asians. J Investig Dermatol. 2016;136(5):1053-1056.

20. Verdin A, Cazier F, Fitoussi R, et al. An in vitro model to evaluate the impact of environmental fine particles (PM0.3-2.5) on skin damage. Toxicol Lett. 2019;305:94-102.

21. Shekar SN, Luciano M, Duffy DL, Martin NG. Genetic and environmental influences on skin pattern deterioration. J Investig Dermatol. 2005;125(6):1119-1129.

22. Gunn DA, Rexbye H, Griffiths CEM, et al. Why some women look young for their age. PLoS One. 2009;4(12):e8021.

23. Nkengne A, Bertin C, Stamatas G, et al. Influence of facial skin attributes on the perceived age of Caucasian women. J Eur Acad Dermatol Venereol. 2008;22(8):982-991.

24. Nkengne A, Stamatas G, Bertin C. Facial skin attributes and age perception. In: Farage MA, Miller KW, Maibach $\mathrm{HI}$ eds. Textbook of Aging Skin. Springer-Verlag;973-980.

25. Samson N, Fink B, Matts PJ. Visible skin condition and perception of human facial appearance: skin and perception of facial appearance. Int J Cosmet Sci. 2009;32(3):167-184.

26. Merinville E, Grennan GZ, Gillbro JM, Mathieu J, Mavon A. Influence of facial skin ageing characteristics on the perceived age in a Russian female population. Int J Cosmet Sci. 2015;37(Suppl 1):3-8.

27. Johnson JM, Kellogg DL. Local thermal control of the human cutaneous circulation. J Appl Physiol (1985). 2010;109(4):1229-1238.

28. Decorps J, Saumet JL, Sommer P, Sigaudo-Roussel D, Fromy B. Effect of ageing on tactile transduction processes. Ageing Res Rev. 2014;13:90-99.

29. Fink B, Grammer K, Matts P. Visible skin color distribution plays a role in the perception of age, attractiveness, and health in female

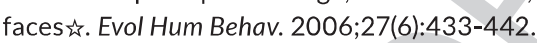

30. Matts PJ, Fink B, Grammer K, Burquest M. Color homogeneity and visual perception of age, health, and attractiveness of female facial skin. J Am Acad Dermatol. 2007;57(6):977-984.
31. Fink B, Matts P. The effects of skin colour distribution and topography cues on the perception of female facial age and health. $J$ Eur Acad Dermatol Venereol. 2008;22(4):493-498.

32. Stamatas GN, Zmudzka BZ, Kollias N, Beer JZ. Non-invasive measurements of skin pigmentation in situ. Pigment Cell Res. 2004;17(6):618-626

33. Minson CT, Berry LT, Joyner MJ. Nitric oxide and neurally mediated regulation of skin blood flow during local heating. J Appl Physiol. 2001;91(4):1619-1626.

34. Stephens DP, Charkoudian N, Benevento JM, Johnson JM, Saumet $\mathrm{JL}$. The influence of topical capsaicin on the local thermal control of skin blood flow in humans. Am J Physiol Regul Integr Comp Physiol. 2001;281:894-901.

35. Holowatz LA. Aging and the control of human skin blood flow. Front Biosci. 2010;15(1):718.

36. Metzler-Wilson K, Kellie LA, Tomc C, Simpson C, Sammons D, Wilson TE. Differential vasodilatory responses to local heating in facial, glabrous and hairy skin. Clin Physiol Funct Imaging. 2012;32(5):361-366.

37. Fiedler J, Grönniger E, Pfanne A, et al. Identification of miR-126 as a new regulator of skin ageing. Exp Dermatol. 2017;26(3):284-286.

38. Shehadeh LA, Webster KA, Hare JM, Vazquez-Padron RI. Dynamic regulation of vascular myosin light chain (MYL9) with injury and aging. PLoS One. 2011;6(10):e25855.

39. Hatake K, Kakishita E, Wakabayashi I, Sakiyama N, Hishida S. Effect of aging on endothelium-dependent vascular relaxation of isolated human basilar artery to thrombin and bradykinin. Stroke. 1990;21(7):1039-1043.

40. Zanetti M, Cappellari GG, Burekovic I, Barazzoni R, Stebel M, Guarnieri G. Caloric restriction improves endothelial dysfunction during vascular aging: effects on nitric oxide synthase isoforms and oxidative stress in rat aorta. Exp Gerontol. 2010;45(11):848-855.

\section{SUPPORTING INFORMATION}

Additional supporting information may be found online in the Supporting Information section.

How to cite this article: Guéré C, Bigouret A, Nkengne A, et al. In elderly Caucasian women, younger facial perceived age correlates with better forearm skin microcirculation reactivity. Skin Res Technol. 2021;00:1-10. https://doi. org/10.1111/srt.13080 